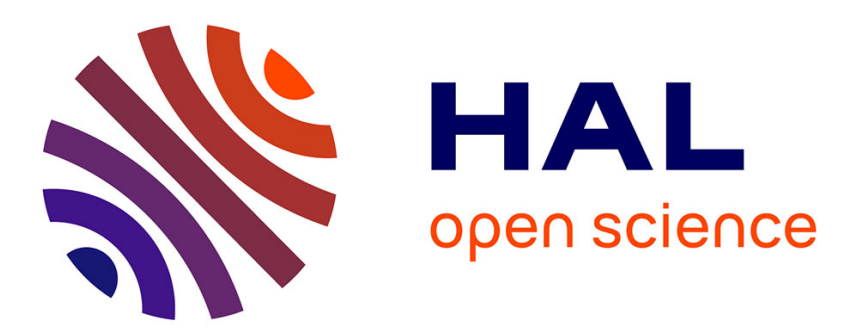

\title{
Feasibility Study of the Elaboration of a Biodegradable and Bioactive Ligament Made of Poly( $\epsilon$-caprolactone)-pNaSS Grafted Fibers for the Reconstruction of Anterior Cruciate Ligament: In Vivo Experiment
}

\author{
A. Leroux, E. Maurice, Véronique Viateau, V. Migonney
}

\section{To cite this version:}

A. Leroux, E. Maurice, Véronique Viateau, V. Migonney. Feasibility Study of the Elaboration of a Biodegradable and Bioactive Ligament Made of Poly $(\epsilon$-caprolactone)-pNaSS Grafted Fibers for the Reconstruction of Anterior Cruciate Ligament: In Vivo Experiment. Innovation and Research in BioMedical engineering, 2019, 40 (1), pp.38-44. 10.1016/j.irbm.2018.10.011 . hal-02397606

\author{
HAL Id: hal-02397606 \\ https://hal.science/hal-02397606
}

Submitted on 6 Dec 2019

HAL is a multi-disciplinary open access archive for the deposit and dissemination of scientific research documents, whether they are published or not. The documents may come from teaching and research institutions in France or abroad, or from public or private research centers.
L'archive ouverte pluridisciplinaire HAL, est destinée au dépôt et à la diffusion de documents scientifiques de niveau recherche, publiés ou non, émanant des établissements d'enseignement et de recherche français ou étrangers, des laboratoires publics ou privés. 
4 Feasibility study of the elaboration of a biodegradable and bioactive ligament anterior cruciate ligament: in vivo experiment

$9 \quad$ Amélie Leroux $^{1 *}$, Emeline Maurice $^{2}$, Véronique Viateau $^{2}$, Véronique Migonney ${ }^{1}$

10

11

$17 *$ Corresponding author

18

E-mail: amelie.leroux@univ-paris13.fr (AL)

${ }^{1}$ Laboratory of Biomaterials and Polymers of Specialty, CSPBAT, UMR CNRS 7244, Institut Galilée, Université Paris 13, Sorbonne Paris Cité, Villetaneuse, France

${ }^{2}$ Ecole Nationale Vétérinaire d'Alfort, Maisons-Alfort, France

9 


\section{Introduction} Injuries of knee anterior cruciate ligament $(A C L)$ frequently happen during high demand sports and

Background - The anterior cruciate ligament rupture is a common injury which mainly affects young and active population. Faced to this problem, the development of synthetic structures for ligament reconstruction is increasing. The most recent researches focused on the development of biodegradable structures that could be functionalized to enhance host integration. This work describes the elaboration of different poly( $\varepsilon$-caprolactone) prototypes for the rat anterior cruciate ligament replacement in order to found the best design for further in vivo assays.

Methods - According to the literature, it was decided to elaborate two different poly( $\varepsilon$-caprolactone) prototypes: a braided one and a free-fibers one. A chemical grafting of a bioactive polymer poly(sodium styrene sulfonate) - was performed on both prototypes and mechanical and biological testing were assessed. Based on these results, one rat was implanted with the best prototype.

Results - The mechanical and biological results demonstrated that the best prototype to implant was the poly(sodium styrene sulfonate)-grafted braided prototype. After one-month implantation, no inflammation was observable around the scar. The rat demonstrated good flexion and extension of the lower limb without any anterior drawer. The prototype was highly anchored to the bone. ESEM images of the explanted prototype showed the presence of cells and tissue ingrowth along and around the fibers.

Conclusion - This work demonstrates the feasibility to implant a bioactive and biodegradable synthetic ligament in the rat model without any inflammation and with a good tissue anchoring at a short-term time. This will lead to an extensive in vivo assay.

Keys words - synthetic ligament; ACL reconstruction; bioactive surface; biodegradable polymer mainly affects young and feminine population[1]. Surgical reconstruction is most often required to

\section{Abstract}


treat the instability of the knee associated with $\mathrm{ACL}$ rupture and until now, the gold standard remains autograft[2]. Nevertheless, side effects as morbidity of autologous tissues, weak anchorage and recurrent pains of the patients led to artificial ligament solutions. The development of synthetic ligament began in the early 1980's with the used of inappropriate materials which lead to synovitis, prosthesis rupture and bone tunnel enlargement[3]. After several clinical fails, the use of poly(ethylene terephthalate) (PET) material was broadly accepted and the current prosthesis on the market, as the LARS ligament, are still made of polyester[4]. Nevertheless, nowadays these synthetic ligaments still encounter limitations such as the anchorage to the bone, the release of tear particles due to abrasion and a poor cell recolonization with limited tissue ingrowth[5]. Due to that, studies are conducted with the purpose of finding a material and design exhibiting similar mechanical properties than the native $A C L$ and being well-integrated in the body avoiding adverse reactions thanks for instance to a surface treatment[6]-[8]. More recent researches focused on the development of biodegradable structures that can support mechanical stresses during the time that a neo-ligament is formed and recovers it first function of stabilizer. So that, the challenge is to found the best polymer to use. Poly(E-caprolactone) (PCL) seems to be promising since it is FDA approved and widely used in different medical devices (MD) applications [9]-[12]. Moreover, our team has shown that the functionalization of PCL films by poly(sodium styrene sulfonate) (pNaSS) grafting can improve the cell response[13]. The development of a new pNaSS-grafted PCL synthetic ligament seems to be relevant as an innovative biodegradable and bioactive ligament prosthesis. In this context, this article aims to compare two different structures of $\mathrm{PCL}$ prototypes for the rat $\mathrm{ACL}$ replacement in order to found the best design for further in vivo assays. To achieve this goal, two different prototypes - braided or composed of free-fibers - were elaborated. After grafting pNaSS on the braided and free fibers prototypes, they were mechanically tested and in vitro assays were conducted. These preliminary data allowed us to determine which structure was the best to be implanted in rat. After one month of in vivo implantation the comportment of this new biodegradable and bioactive prosthesis was analyzed. It was expected that the braided pNaSS- 
grafted prototype was still present after one month implantation, induce cell colonization and be a support for rat walk.

\section{Material and methods}

\section{Preparation of PCL prototypes}

20 PCL fibers (diameter $110 \pm 15 \mu \mathrm{m}$, length $75 \pm 5 \mathrm{~mm}$ ) from Luxilon company (LUXILON Industries nv, Antwerpen, Belgium) are associated and hand tied in order to achieve 20 fibers PCL bundles. PCL bundles are then used to elaborate to kinds of ligament prototypes: (i) a "braided prototype" made of fully braided bundles, (ii) a "free-fibers prototype" which is partially braided at its two end portions around a central free fiber part (see Fig1).

Whatever the prototype, it is divided in two parts: (1) an implanted part (15 $\pm 2 \mathrm{~mm}$ long) which is inserted in the knee articulation of the rat (intra articular and osseous parts) and (2) an extra part ( $30 \pm 5 \mathrm{~mm}$ long) which allows the veterinarian to optimally implant the ligament into the knee joint and straighten it for the best stabilization - this part is cut at the end of the implantation. Once the prototypes are made, they are ultrasound washed with different solvents $(10 \%(\mathrm{w} / \mathrm{v}))$ in order to remove the spin finish as following: hexane for $15 \mathrm{~min}$, absolute ethanol for $5 \mathrm{~min}$, water two times for $5 \mathrm{~min}$. Samples are then dried under vacuum and stored at $4^{\circ} \mathrm{C}$ until grafting process or experiments.

\section{Grafting of poly(sodium styrene sulfonate) on PCL prototypes}

PCL prototypes are functionalized with poly(sodium styrene sulfonate) pNaSS using a grafting "from" technique [14]. After the spin finish removal step, $\mathrm{PCL}$ samples are ozonized at $30^{\circ} \mathrm{C}$ in distilled water under stirring for $10 \mathrm{~min}$. Ozone is generated using an ozone generator BMT $802 \mathrm{~N}$ (ACW) with a gas pressure of 0.5 bars and an oxygen flow rate of $0.6 \mathrm{~L} \mathrm{~min}^{-1}$. Secondly, the ozonized PCL samples are transferred into a degassed aqueous NaSS solution $(15 \%(\mathrm{w} / \mathrm{v}))$ under argon and maintained 1 hour at $45^{\circ} \mathrm{C}$ under stirring to allow the radical polymerization of the monomer. Then, samples are extensively washed with distilled water for 48 hours and vacuum-dried. The evidence of the pNaSS 
grafting is provided by toluidine blue colorimetric assay and the grafting rate is determined according to Ciobanu et al[14].

\section{Mechanical testing}

The two prototypes have undergone tensile loading until rupture by using a Bose Electroforce 3230 equipment (Bose). The tests were carried out three times for each prototype - effective length of $18.8 \pm 1.1 \mathrm{~mm}$ - the jaws of the machine were placed after the implanted part to test the future implant in its entirety. Stress strain curves are recorded and maximum force $\left(F_{\max }\right)$ in $N$, ultimate tensile stress (UTS) in MPa and elongation $(\varepsilon)$ in \% are determined.

\section{Sterilization}

Before biological experiments all the prototypes were packaged and sterilized as following: two washing of 3 hours in $1.5 \mathrm{M}$ aqueous sodium chloride solution; one washing of $10 \mathrm{~min}$ in ultra-pure water; two washing of 3 hours in $0.15 \mathrm{M}$ aqueous sodium chloride solution; one washing of $10 \mathrm{~min}$ in ultra-pure water; one washing of 3 hours in DPBS (Gibco); 20 minutes in 70\% ethanol solution; following by 10 min ultra-pure water bath; then 15 min of UV irradiation were applied in both sides of the samples. All the washing was done under stirring. Prototypes were kept in sterile PBS solution at $4^{\circ} \mathrm{C}$ until experiments.

\section{Biological experiments}

\section{Cell isolation}

Anterior cruciate ligaments (ACL) from sheep are collected thanks to the collaboration with the ENVA (Maisons-Alfort, France (Ligart protocol)). Tissues are cut into small pieces of 1 to $2 \mathrm{~mm}^{2}$, washed three times in DPBS (Gibco) and incubated in a $0.1 \%(\mathrm{w} / \mathrm{v})$ collagenase (Sigma-Aldrich) solution for 6 hours at $37^{\circ} \mathrm{C}$ under $5 \% \mathrm{CO}_{2}$. The mixture solution is centrifuged ( $3 \mathrm{~min}$ at $1500 \mathrm{rpm}$ ): the supernatant is withdrawn and the clot is suspended in DMEM (Gibco) complemented with $10 \%$ 

fibroblasts are cultured in flask until confluence and prior seeding on samples.

\section{In vitro study}

pNaSS-grafted and ungrafted "free-fibers" and "braided" prototypes are placed in a 6-wells plate treated for low attachment cell cultures. A primary fibroblasts suspension (P3) of 300000 cells in $50 \mu \mathrm{L}$ are seeded onto the middle of the implanted part of the different prototypes and incubated 30 minutes at $37^{\circ} \mathrm{C}$ under $5 \% \mathrm{CO}_{2}$, then $200 \mu \mathrm{L}$ of medium are added for $1 \mathrm{~h}$ to limit the drying. Each well was then completed with $2 \mathrm{~mL}$ of $10 \%$ serum supplemented DMEM and cultured for 1 month (33 days) at $37^{\circ} \mathrm{C}$ with a fresh medium replacement three times per week. After this period of time, the prototypes were fixed 1 hour in a $4 \%$ formaldehyde solution, intensively rinse with ultra-pure water, and observed in an environmental scanning electron microscope (ESEM). ESEM images were carried out using a Hitachi TM3000 SEM operating at $15 \mathrm{kV}$ and equipped with a Peltier cooling device running at $4^{\circ} \mathrm{C}$.

\section{In vivo study}

A 37-weeks-old female Wistar rat of 360g (approved supplier Janvier Labs, Saint Berthevin, France) was implanted with a pNaSS-grafted braided prototype. Anesthesia was performed by intraperitoneal injection of a mixture of dexmedetomidine/ketamine $(0.2 \mathrm{mg} / \mathrm{kg}$ and $80 \mathrm{mg} / \mathrm{kg}$ respectively, Domitor ${ }^{\circledR}$ Orion pharma and Imalgene ${ }^{\circledR}$ Merial) and then maintained by inhalation of an isoflurane/O2 mixture. The surgical procedure was then performed on the lower left limb. After exposition of the ACL by arthrotomy, the ligament was excised. Then, the knee was oriented in a hyper-flexion position and the femur and the tibia were drilled at a diameter of 1.5 and $1.1 \mathrm{~mm}$ respectively, the prototype was placed in the articulation through a $1.2 \mathrm{~mm}$ diameter needle, replacing the native ACL. Screws of $6 \mathrm{~mm}$ (femur) and $7 \mathrm{~mm}$ (tibia) length (references VS112.006 and VS112.007, Synthes) were placed for the fixation of the prototype into the bone. The prototype surplus was cut flush to not exceed the joint. The incision was closed in three stages: (i) closure of the 
143 joint capsule by interlocking with U-shaped stitches (Glycomère 631, Biosyn 1.5 decimal, Covidien);

144 (ii) closure of the subcutaneous connective tissue by a simple suture (glycomère 631, Biosyn 1

145 decimal, Covidien) ; (iii) closure of the skin by intradermal overlock (glycomère 631, Biosyn 1 decimal,

146 Covidien). No restriction on movements was applied. After one month implantation, the rat was

147 sacrificed and the prototype was explanted going back through the osseous tunnel. The explant was

148 fixed 1 hour in $4 \%$ formaldehyde solution and observed with ESEM-Peltier at $4^{\circ} \mathrm{C}$.

\section{Results}

\section{Elaboration of prototypes}

151

152

153

154

155

The free fibers and the braided prototypes were observed microscopically in order to validate that the handmade elaboration did not alter the PCL fibers. As it can be seen in the Figure 1 , the fibers do not look impacted by the assembly.

Figure 1 : SEM images of free fibers (A) and braided (B) prototypes

The presence of grafted pNaSS molecules on PCL prototypes was determined according to the toluidine blue assays process. The obtained average value of the grafting rate of the free fibers prototypes $(n=3)$ equals $0.70 \pm 0.12 \mu$ mol. $\mathrm{g}^{-1}$ whereas the grafting rate of braided prototypes $(n=3)$ reached $1.05 \pm 0.14 \mu \mathrm{mol} . \mathrm{g}^{-1}$ (see. Fig.2).

Figure 2 : Grating rates of the two types of prototypes obtained with the toluidine blue colorimetric method

\section{Mechanical testing}

Because the prototypes are handmade elaborated, the presence of some knots was necessary in order to demarcate the braided areas from the free fibers areas, especially at the intra-articular part level for free-fibers prototypes and for the limit between implanted and the external parts for both prototypes. To avoid the compression of the knot in the jaws and so influence the test, it was decided to evaluate the mechanical properties of the prototypes by placing the jaws after the implanted area (see.Fig.3).

Figure 3 : schematic representation of the placement of the prototypes in the jaws (in gray) of the traction machine 
The mechanical results and the location of the rupture are presented in the Table 1 . The obtained results showed that the "free fibers" prototypes exhibit lower mechanical properties than braided prototypes with a maximal force of $38.8 \pm 3.7 \mathrm{~N}$ against $43.3 \pm 1.0 \mathrm{~N}$ and an UTS of $204.3 \pm 19.4 \mathrm{MPa}$ against 227.9 $\pm 5.4 \mathrm{MPa}$ that can be endured. Moreover, the elongation of the braided prototypes is 1.5 time more important than the one of the free fibers prototypes going from $77.5 \pm 9.8 \%$ to $115.6 \pm 4.6 \%$. Finally, it is important to note that it was observed that the rupture always came above or below a knot and not in the middle of the implanted part of the prototype. The prototypes are therefore weakened by the presence of these knots.

\begin{tabular}{lllll}
\hline & $\mathbf{F}_{\max } \mathbf{( N )}$ & UTS (MPa) & $\boldsymbol{\varepsilon}$ (\%) & Location of the rupture \\
\hline Free fibers prototypes & & & & \\
\#1 & 40.95 & 215.45 & 88.22 & above knot 1 \\
\#2 & 40.95 & 215.45 & 75.00 & below knot 1 \\
\#3 & 34.57 & 181.88 & 69.15 & below knot 1 \\
Average & $\mathbf{3 8 . 8 \pm 3 . 7}$ & $\mathbf{2 0 4 . 3 \pm 1 9 . 4}$ & $\mathbf{7 7 . 5 \pm 9 . 8}$ & \\
\hline Braided prototypes & & & & \\
\#1 & 42.24 & 222.24 & 110.29 & below knot 2 \\
\#2 & 43.38 & 228.45 & 118.74 & above knot 2 \\
\#3 & 44.29 & 233.02 & 117.74 & above knot 2 \\
\hline Average & $\mathbf{4 3 . 3 \pm 1 . 0}$ & $\mathbf{2 2 7 . 9 \pm 5 . 4}$ & $\mathbf{1 1 5 . 6 \pm 4 . 6}$ & \\
\hline
\end{tabular}

Table 1 : Mechanical properties of the two PCL prototypes

\section{Biological experiments}

\section{Cell culture in vitro assays}

The observation of the cell cultures after 1 month revealed differences on the cells repartition when there are seeded onto the free fibers prototypes when compared to the braided prototypes (see Figure 4 and 5).

Figure 4 : ESEM images of ungrafted $(A, B, C)$ and grafted $(D, E, F)$ free fibers prototypes cultured with primary fibroblasts during 1 month.

Figure 5 : ESEM images of ungrafted $(A, B, C)$ and grafted $(D, E, F)$ braided prototypes cultured with primary fibroblasts during 1 month.

With the free fibers prototypes, it can be seen that the cells mainly grow between the fibers and that there are quite separated one from each other (see Fig.4). The ungrafted free fibers prototype presents fewer cells that the pNaSS grafted one (see Fig.4.B and 4.E) and the cells are better spread with the pNaSS grafting (see Fig.4.C and 4.F). 
The ESEM images suggest that there are more cells that have grown onto the braided prototypes when compared to the free fibers prototypes (see Fig.4.A and 5.A). Moreover, with the braided prototypes, the cells also grow between the fibers but they seem to be more inter-connected starting to form a cellular network. This fact is clearly enhanced by the presence of the pNaSS grafting (see Fig.5.C and 5.F). When we compare the ungrafted and the grafted braided prototypes, it can be observed that there are numerically more cells with the pNaSS is present on the fibers (see. Fig.5.A and 5.D); in addition cells are better spread and they started to coat the fibers all around (see Fig.5.C and 5.F).

\section{In vivo assay}

\section{Clinical evidences}

The day after the implantation, the rat was clearly using it limb, no evidence of lameness was noted during the implantation period. After one month, the $360 \mathrm{~g}$-female Wistar rat was sacrificed. The rat was weighted and an increase of $15 \mathrm{~g}$ was noticed. The observation of the animal showed a slight swelling of the implanted paw with respect to the opposite limb, accompanied by a slight edema but without any sign of inflammation (see Fig.6). The range of motion of the joint was normal, with good flexion and good extension, and with no anterior drawer, compared to the non-operated contralateral limb. The joint was exposed and the implanted pNaSS-grafted braided prototype was explanted. The prototype was difficult to withdraw because of it good stability and anchorage in the osseous tunnels.

Figure 6 : implanted limb of the rat (A) and prototype implanted (B) after 1 month

\section{Explant observations}

After cells and tissue fixation, the braided prototype was observed with ESEM. After 1 month implantation, we can see that the prototype has been recolonized by tissue (see Fig. 7). The recolonization is more important in the osseous part compared to the intra-articular part (see Fig.7.A and 7.C), probably thanks to the close proximity of cells. In both intra articular and osseous parts, the fibers are well surrounded by tissue. The image of the cross section of the prototype (see. Fig.7.B) 
shows the presence of tissue between the fibers confirming the good integration of the pNaSS-

217 grafted braided prototype in the articulation.

Figure 7 : ESEM images of the pNaSS-grafted braided prototype. A and B present the intra-articular part; C and D focus on the intra-osseous part.

\section{Discussion}

Based on the literature, two types of prototypes were designed in order to found the best structure for the reconstruction of the anterior cruciate ligament in the rat model: (1) a free fibers prototype clearly inspired by the LARS ligament[15] and (2) a braided prototype which is an option that several scientists try to explore[16], [17].

The covalent grafting of a bioactive polymer -poly(sodium styrene sulfonate) - has been developed by our team onto PCL films and its bioactive effect has already been demonstrated[13]. In this study, it is shown that the grafting process can be extent to fibers structure, with however a slightly better grafting rate onto the braided prototypes.

The mechanical results obtained for both prototypes clearly demonstrate the limit of handmade structure which required knots to stay united. In fact, these knots are going to concentrate stresses and so weaken the prototypes. The more knots there are, the more fragile is the structure. Moreover, in the case of the reconstruction of $A C L$ in Wistar rat, the literature reports a failure load of the native $A C L$, for rats that weight between $300 \mathrm{~g}$ and $485 \mathrm{~g}$, of 34.5-35.5 N depending of the weight but also of the age of the animal[18], [19]. In our case, it was mechanically safer to choose to implant the PCL braided prototype which can endure a maximal force of $43.3 \pm 1.0 \mathrm{~N}$ before failure.

The in vitro study on both grafted and ungrafted free fibers and braided prototypes was done. The previously described bioactive feature can be found again in a prototype structure with a proliferation enhancement and a more homogenous repartition of cells thanks to the presence of pNaSS. The in vitro results demonstrated clearly that the pNaSS-grafted braided prototype is the best candidate for bioactive and biodegradable ligament prosthesis in rat.

Because this study was mainly conducted to evaluate the surgical feasibility to implant anterior cruciate ligament prosthesis in rat before starting an extensive in vivo study, only one rat was 
implanted. According to the previous results, it is obvious that our choice go to the pNaSS-grafted braided prototype. Despite the small size of the animal and the need of very precise material, the prototype was successfully implanted. After one month implantation, good clinical outcomes were noticed with among other no lameness, no inflammation and no anterior drawer. Finally, the observation of the explanted prototype demonstrates the potential of this implant which has been recolonized both at the intra-osseous and intra-articular level and around and along the fibers.

\section{Conclusion}

This study validates the feasibility to implant a bioactive and a biodegradable ligament to replace the anterior cruciate ligament in the rat model, at least at the short-term. Our future works are going to be concentrated to design a braided or knitted structure that does not imply the use of knots and that can be standardized. Once this new structure will be operational, the implantation of numerous rats in the context of an extensive in vivo study, which will compare the grafted and the ungrafted prosthesis, will be conducted.

\section{Acknowledgments}

The authors would like to thank Pr V. Viateau (ENVA, Maisons-Alfort, France) for the surgical implantation ; Jade Chura for technical help in the mechanical part and Herbert Debreuck from Luxilon company (LUXILON Industries nv, Antwerpen, Belgium) for providing PCL fibers. This work was funded as part of the "Future Investment Project" by the French Public Investment Bank and the French state - PSPC application - Liga2bio project.

\section{References}

[1] D. E. Gwinn, J. H. Wilckens, E. R. McDevitt, G. Ross, and T.-C. Kao, "The Relative Incidence of Anterior Cruciate Ligament Injury in Men and Women at the United States Naval Academy," Am. J. Sports Med., vol. 28, no. 1, pp. 98-102, Jan. 2000.

[2] T. Jamil, U. Ansari, M. Najabat Ali, and M. Mir, "A Review on Biomechanical and Treatment Aspects Associated with Anterior Cruciate Ligament," IRBM, vol. 38, no. 1, pp. 13-25, Feb. 2017.

[3] P. A. Indelicato, M. S. Pascale, and M. O. Huegel, "Early experience with the GORE-TEX polytetrafluoroethylene anterior cruciate ligament prosthesis," Am. J. Sports Med., vol. 17, no. 1, pp. 55-62, Jan. 1989. 
[4] M.-F. Guidoin, Y. Marois, J. Bejui, N. Poddevin, M. W. King, and R. Guidoin, "Analysis of retrieved polymer fiber based replacements for the ACL," Biomaterials, vol. 21, no. 23, pp. 2461-2474, Dec. 2000.

[5] A. Ventura, C. Terzaghi, C. Legnani, E. Borgo, and W. Albisetti, "Synthetic grafts for anterior cruciate ligament rupture: 19-year outcome study," The Knee, vol. 17, no. 2, pp. 108-113, Mar. 2010.

[6] C. T. Laurencin and J. W. Freeman, "Ligament tissue engineering: An evolutionary materials science approach," Biomaterials, vol. 26, no. 36, pp. 7530-7536, Dec. 2005.

[7] C. Vaquette et al., "The effect of polystyrene sodium sulfonate grafting on polyethylene terephthalate artificial ligaments on in vitro mineralisation and in vivo bone tissue integration," Biomaterials, vol. 34, no. 29, pp. 7048-7063, Sep. 2013.

[8] V. Viateau, M. Manassero, F. Anagnostou, S. Guérard, D. Mitton, and V. Migonney, "Biological and Biomechanical Evaluation of the Ligament Advanced Reinforcement System (LARS AC) in a Sheep Model of Anterior Cruciate Ligament Replacement: A 3-Month and 12-Month Study," Arthrosc. J. Arthrosc. Relat. Surg., vol. 29, no. 6, pp. 1079-1088, Jun. 2013.

[9] P. Zhao, F. Han, P. Zhang, Y. Sun, C. Lin, and J. Chen, "Hydroxyapatite-doped polycaprolactone nanofiber membrane improves tendon\&ndash;bone interface healing for anterior cruciate ligament reconstruction," Int. J. Nanomedicine, p. 7333, Dec. 2015.

[10] G. Yang, H. Lin, B. B. Rothrauff, S. Yu, and R. S. Tuan, "Multilayered polycaprolactone/gelatin fiber-hydrogel composite for tendon tissue engineering," Acta Biomater., vol. 35, pp. 68-76, Apr. 2016.

[11]A. C. Gurlek, B. Sevinc, E. Bayrak, and C. Erisken, "Synthesis and characterization of polycaprolactone for anterior cruciate ligament regeneration," Mater. Sci. Eng. C, vol. 71, pp. 820-826, Feb. 2017.

[12] G. Wu, X. Deng, J. Song, and F. Chen, "Enhanced biological properties of biomimetic apatite fabricated polycaprolactone/chitosan nanofibrous bio-composite for tendon and ligament regeneration," J. Photochem. Photobiol. B, vol. 178, pp. 27-32, Jan. 2018.

[13] G. Rohman, S. Huot, M. Vilas-Boas, G. Radu-Bostan, D. G. Castner, and V. Migonney, "The grafting of a thin layer of poly(sodium styrene sulfonate) onto poly( $\varepsilon$-caprolactone) surface can enhance fibroblast behavior," J. Mater. Sci. Mater. Med., vol. 26, no. 7, Jul. 2015.

[14] M. Ciobanu, A. Siove, V. Gueguen, L. J. Gamble, D. G. Castner, and V. Migonney, "Radical graft polymerization of styrene sulfonate on poly (ethylene terephthalate) films for $A C L$ applications:'grafting from' and chemical characterization," Biomacromolecules, vol. 7, no. 3, pp. 755-760, 2006.

[15] G. Dericks, "Ligament advanced reinforcementsystem anterior cruciate ligament reconstruction," Oper. Tech. Sports Med., vol. 3, no. 3, pp. 187-205, Jul. 1995.

[16] J. W. Freeman, M. D. Woods, and C. T. Laurencin, "Tissue engineering of the anterior cruciate ligament using a braid-twist scaffold design," J. Biomech., vol. 40, no. 9, pp. 2029-2036, Jan. 2007.

[17]S. Sahoo, J. G. Cho-Hong, and T. Siew-Lok, "Development of hybrid polymer scaffolds for potential applications in ligament and tendon tissue engineering," Biomed. Mater., vol. 2, no. 3, pp. 169-173, Sep. 2007.

[18] F. A. Petrigliano, G. A. Arom, A. N. Nazemi, M. G. Yeranosian, B. M. Wu, and D. R. McAllister, "In Vivo Evaluation of Electrospun Polycaprolactone Graft for Anterior Cruciate Ligament Engineering," Tissue Eng. Part A, vol. 21, no. 7-8, pp. 1228-1236, Apr. 2015.

[19] W. A. Romani, S. M. Belkoff, and J. H. Elisseeff, "Testosterone may increase rat anterior cruciate ligament strength," The Knee, vol. 23, no. 6, pp. 1069-1073, Dec. 2016. 